\title{
Körperwahrnehmung bei Rückenschmerzen
}

\author{
Hannu Luomajoki und Fritz Zahnd
}

Aktuelle Studien belegen Körperwahrnehmungsprobleme bei Phantomschmerzen, CRPS und beim Karpaltunnelsyndrom. Leiden Patienten mit Rückenschmerzen ebenfalls unter Störungen der Körperwahrnehmung? Wie kann man solche Symptome testen, und mit welchen Übungen diesem Phänomen begegnen? Der folgende Artikel bietet praxisnahe Antworten auf diese Fragen.

Die Körperwahrnehmung findet im sensorischen Kortex statt. Ein sogenannter Homunkulus zeigt in Form eines kleinen Männchens die Proportionen der verschiedenen Körperareale entsprechend deren Repräsentation auf der Gehirnrinde auf ( $\bullet$ Abb. 1).
Je größer die Repräsentationsareale im sensorischen Homunkulus sind, umso besser ist das Differenzierungsvermögen des Zentralen Nervensystems. Entsprechend werden Areale mit großem Repräsentationsareal sehr gut vom Gehirn wahrgenommen. Dieses Differenzie-

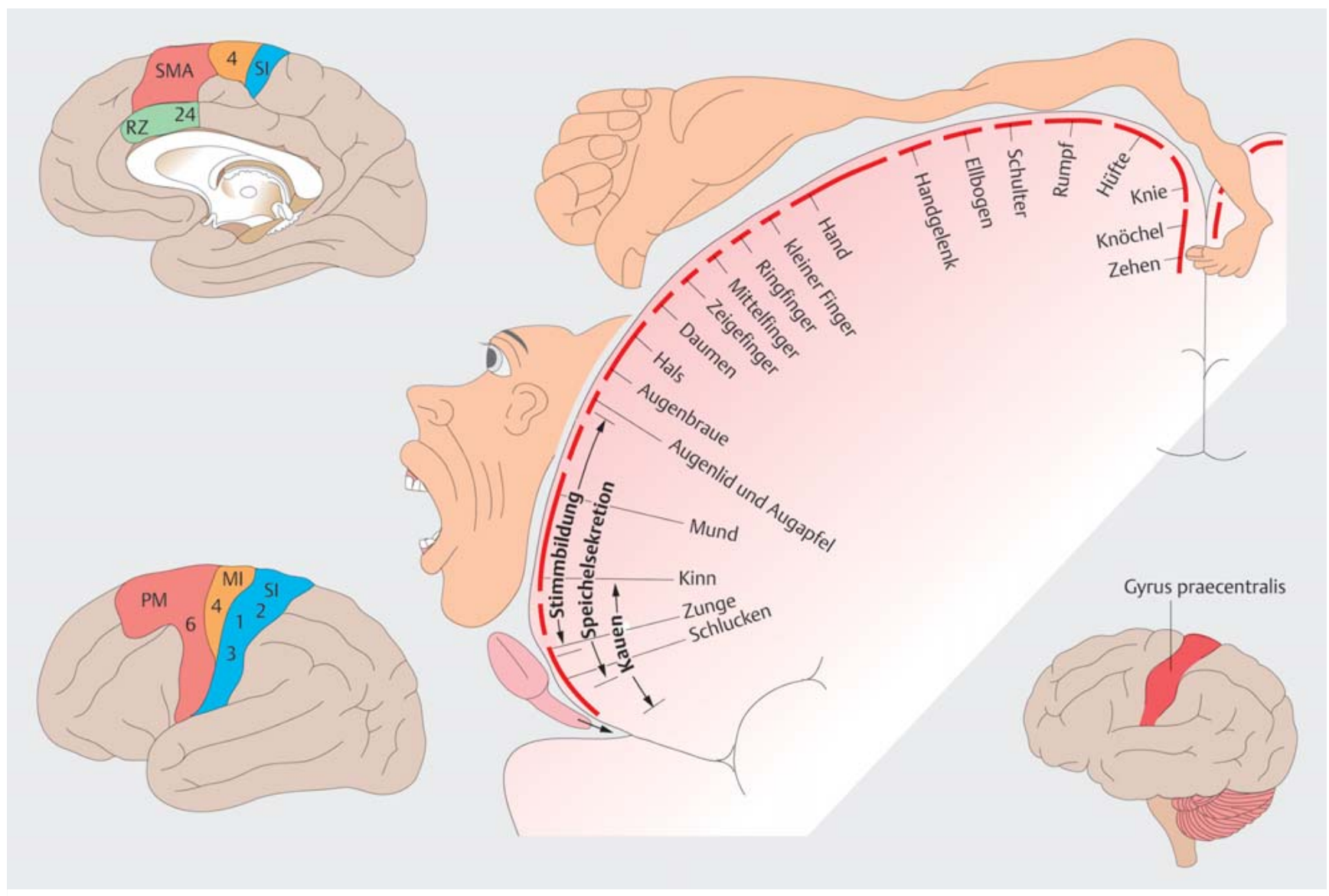

Abb. 1 Sensorischer Kortex mit Homunkulus. Quelle: Thieme Gruppe 
rungsvermögen ist im Gesicht, an Zunge und Lippen sehr gut sowie an den Fingerspitzen am besten ausgeprägt.

Gehirn-Training Die Evolution trainierte das Gehirn. So verbesserte sich die Wahrnehmung in einem Körperteil, je mehr man sich auf diesen konzentrierte und ihn benutzte. Heute können wir mit dem richtigen „Fingerspitzengefühl“ unterschiedlichste Stoffe oder Oberflächen erkennen, Zunge und Nase nehmen verschiedenste Geschmacksrichtungen bis hin zu Pheromonen wahr. Demgegenüber belegen andere Körperregionen wie die LWS oder das Knie nur kleine Bereiche im sensorischen Kortex. Die Wahrnehmung dieser Regionen erfolgt merklich undifferenzierter als die Sinneswahrnehmung von Fingern oder Händen. Wenn es also um den eigenen Rücken geht, können wir lediglich sagen, ob sich dieser „verspannt“ anfühlt.

Das Gehirn arbeitet - wie dies auch von der Muskulatur bekannt ist - getreu dem Motto „Use it or lose it!“. Im Klartext: Ein viel benutzter Körperbereich wird kortikal gut repräsentiert. Gleichzeitig sprechen einige Indizien dafür, dass sich ein sensorisches Repräsentationsareal durch Schmerzen verändert.

\section{Änderung der Körperwahrnehmung}

\section{Phantomschmerzen}

Anhand von Phantomschmerzen wird deutlich, dass Schmerzen die Körperwahrnehmung verändern. In den 1980er-Jahren wurde nachgewiesen, dass das PhantomPhänomen mit der Repräsentation im sensorischen Kortex zusammenhängt [23]. Der Neurowissenschaftler Ramachrandran manipulierte mittels eines Spiegels die Wahrnehmung seiner Patienten: das Betrachten des Spiegelbildes der noch vorhandenen Hand linderte den Phantomschmerz der amputierten Hand.

\section{CRPS und CTS}

Zur Jahrtausendwende wiesen verschiedene Forscher auch bei beim CRPS (Complex Regional Pain Syndrome; vormals: Morbus Sudeck) ähnliche Veränderung im sensorischen Homunkulus nach $[1,7,8,11,21]$. Später wurden erfolgreiche klinische Studien zur Normalisierung dieses Phänomens durchgeführt [17, 18,22]: Moseley konnte Schmerz und Schwellung einer CRPS-Hand durch eine Kombination aus Laterality Recognition, Imaginary Movements sowie Spiegelbox-Übungen reduzieren [17].

Eine Studie aus dem Jahre 2012 zeigt, dass selbst beim schmerzfreien Karpaltunnelsyndrom (CTS) die Körperwahrnehmung gestört zu sein scheint [25]. Beim Laterality-Recognition-Test erkannten die Forscher signifikante Unterschiede der Körperwahrnehmung von gesunder und betroffener Seite.

\section{Test der Körperwahrnehmung}

Die nachfolgend vorgestellten Testverfahren wurden an der Lendenwirbelsäule untersucht. Im Fokus stand dabei zumeist der chronische nicht-spezifische Rückenschmerz (Chronic Non Specific Low Back Pain bzw. CNSLBP).

\section{MÖGLICHKEITEN ZUM TESTEN DER KÖRPERWAHRNEHMUNG \\ - Zwei-Punkte-Diskrimination (TPD) \\ - Laterality-Recognition-Test \\ - Zeichnen des eigenen Rückens \\ - Stimulus-Lokalisation \\ - Motor-Control-Impairment (MCI)}

\section{Zwei-Punkte-Diskrimination}

Das für die Untersuchung und Behandlung der Hand etablierte Verfahren der Zwei-Punkte-Diskrimination (Two-Point-Discrimiation, TPD) wurde für die LWS adaptiert $[15,16]$. Unter Verwendung einer Schublehre wurde die Schwelle des Erkennens zweier auseinander liegender Kontaktpunkte auf der Rückenhaut zwischen erstem Lendenwirbel und Beckenkamm in horizontaler und vertikaler Richtung gemessen ( $\bullet$ Abb. 2).

Die Messung erfolgte mit konstantem Druck auf die Haut des Patienten in $5 \mathrm{~mm}$-Schritten entweder absteigend von $10 \mathrm{~cm}$ oder aufsteigend von $1 \mathrm{~cm}$. Ab- und aufsteigende Messungen wurden kombiniert, um Ratespiele der Testpersonen auszuschließen. Bei Gesunden lag der durchschnittliche TPD-Wert bei $44 \mathrm{~mm}$, während bei Rückenschmerzpatienten der Wert auf $62 \mathrm{~mm}$ anstieg. Nach Catley können TPD-Werte über 60 mm mit 90\%iger Sicherheit als „abnormal“ bezeichnet werden [3].

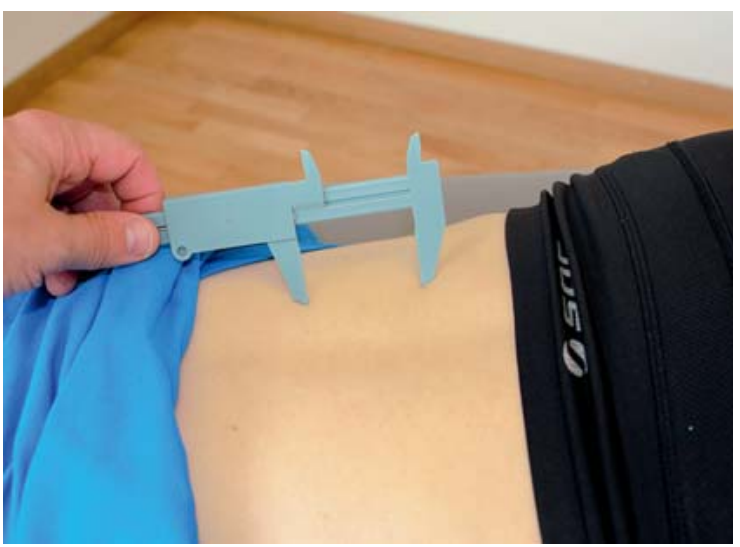

- Abb. 2 Zwei-Punkte-Diskrimination am Rücken. Quelle: Hannu Luomajoki und Fritz Zahnd (nachgestellte Situation). 
- Tab. 1 Testergebnis der Studie „Zeichnen des eigenen Rückens“ mit sechs CNSLBP-Patienten (nach Moseley 2008) [19].

\begin{tabular}{|c|c|c|}
\hline Einzeichnen der Begrenzungslinien des eigenen Rückens & $\begin{array}{l}\text { Einzeichnen der wahrgenommenen } \\
\text { Wirbel }\end{array}$ & Zwei-Punkte-Diskrimination \\
\hline $\begin{array}{l}\text { - Alle Patienten sagten, sie können „die Begrenzung } \\
\text { ihres Rückens nicht finden“. } \\
\text { - Mehrere Patienten pausierten bei der Aufgabe: } \\
\text { „Es fühlt sich an, als sei mein Rücken geschrumpft“. } \\
\text { - Fünf Patienten konnten die Begrenzungslinien nicht } \\
\text { vollständig aufzeichnen. } \\
\text { - Fehlende Begrenzungslinien befanden sich stets auf } \\
\text { Höhe und Seite des Rückenschmerzes. }\end{array}$ & $\begin{array}{l}\text { - Kein Patient zeichnete alle Wirbel ein. } \\
\text { - Eingezeichnete Wirbel wichen ab zur } \\
\text { Seite von fehlender Begrenzungslinie } \\
\text { und Schmerz. } \\
\text { - Fehlende Wirbel befanden sich auf } \\
\text { Höhe fehlender Begrenzungslinien. }\end{array}$ & $\begin{array}{l}\text { - Bei fünf Patienten war die TPD } \\
\text { auf Höhe und Seite der fehlenden } \\
\text { Begrenzungslinie vergrößert. }\end{array}$ \\
\hline
\end{tabular}

\section{Laterality-Recognition-Test}

Um die Links-Rechts-Diskrimination zu beurteilen, wurde „Recognise“ entwickelt. Die Software vom Neuro Orthopaedic Institute (www.noigroup.com) spielt u. a. 56 Bilder eines rotierten männlichen Rumpfes in verschiedenen Positionen randomisiert ab. 28 Aufnahmen zeigen eine Rechtsrotation, 28 eine Drehung nach links.

Seitenerkennung Im Jahre 2009 verglichen Bray und Moseley 21 Patienten mit unspezifischen chronischen Rückenschmerzen mit 14 asymptomatischen Personen [2]. Beide Gruppen erhielten zwei Aufgaben mit jeweils zwei aus 40 Bildern bestehenden Tests. Die Probanden sollten die Fotos dahingehend beurteilen, ob ein Rumpf links- oder rechtsrotiert abgebildet ist. Als Kontrollaufgabe sollten Hände als links- oder rechtsseitig beurteilt werden.

Treffsicherheit Die Analyse beider Tests erfolgte anhand von Antwortzeit und Treffsicherheit. Berücksichtigt wurde, ob die Teilnehmer der Patientengruppe unter unioder bilateralen Rückenschmerzen litten. Während sich die Antwortzeiten für alle Gruppen ähnlich verhielten, war die Treffsicherheit unterschiedlich. So unterliefen bilateralen CNSLBP-Patienten mehr Fehler als Personen mit unilateralen Rückenschmerzen. Diese wiederum beurteilten die Fotos mit der Rumpfrotation schlechter als symptomfreie Personen. Bei den Beurteilungsaufgaben zur Hand wurde dieses Phänomen nicht beobachtet.

Demgemäß eignet sich der Laterality-Recognition-Test, um bei CNSLBP-Patienten eine Störung der Rumpfwahrnehmung festzustellen und um den Behandlungsverlauf zu dokumentieren.

\section{Zeichnen des eigenen Rückens}

In einer früheren Studie von Moseley wurde sechs CNSLBP-Patienten ein Bild mit der schematischen Zeichnung eines Rückens gezeigt [19]. Auf der Grafik waren lediglich die Schulterpartie sowie der untere Teil des Beckens angedeutet. Die Probanden wurden aufgefordert, die Begrenzungslinien ihres Rückens auf der Zeichnung zu ergänzen. Dabei sollten sich die Testpersonen nicht auf das objektive Aussehen ihres Rückens konzentrieren, sondern vielmehr auf die Art und Weise, wie sich dieser anfühlt. Zum zweiten sollten die Probanden die von ihnen wahrgenommenen Wirbel einzeichnen. Zusätzlich wurde die Zwei-Punkte-Diskrimination gemessen. Die Analyse zu den einzelnen Datenerhebungen offenbarte diverse Auffälligkeiten ( Tab. 1).

Zur Körperwahrnehmung des Rückens wurde ein spezieller Fragebogen entwickelt [25]: der Fremantle-BackAwareness-Questionnaire wurde auch in einer deutschen Version validiert [4].

\section{Training der Körperwahrnehmung}

\author{
5 MÖGLICHKEITEN ZUM TRAINING \\ DER KÖRPERWAHRNEHMUNG \\ - Taktiles sensorisches Training und Graphästhesie \\ - Motor Imagery \\ - Laterality-Recognition \\ - Isolierte Low-Load-Anspannung lokaler Muskeln \\ - Dissoziationsübungen
}

\section{Taktiles Sensorisches Training und Graphästhesie}

Taktiles Sensorisches Training bei CRPS-Patienten funktioniert besser, wenn die Betroffenen während des Übens ein Bild des betroffenen Körperareals sehen [20].

Im Rahmen einer Fallstudie mit drei chronischen Rückenschmerzpatienten setze Wand eine Reihe von Übungen zur Körperwahrnehmung in Form eines progressiven Trainings ein [27]. Den Probanden wurden verschiedene taktile Reize am Rücken appliziert - zunächst während der Betrachtung eines Rückenbildes, im zweiten Schritt ohne Bild. Die Testpersonen sollten auf einer Zeichnung jene Punkte markieren, an denen ihr Rücken berührt wurde. Gesteigert wurden die Anforderungen zuerst durch die motorische Vorstellung von Bewegung, später durch 


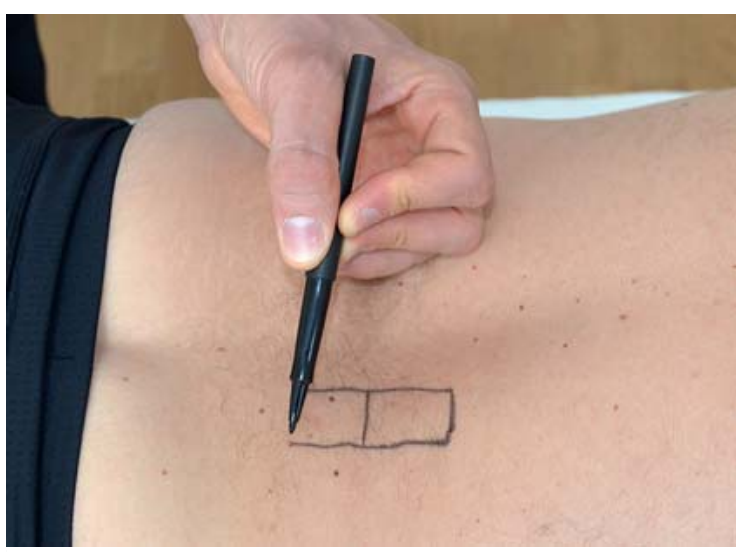

- Abb. 3 Graphästhesie bezeichnet die Fähigkeit, durch Druck „geschriebene“ Buchstaben oder Ziffern auf der Haut zu erkennen, ohne dabei hinzusehen. Voraussetzung hierfür ist eine intakte taktile Wahrnehmung. Quelle: Hannu Luomajoki und Fritz Zahnd (nachgestellte Situation).

dissoziative Übungen. Die Resultate waren sehr gut: nach fünf Monaten waren alle Patienten beschwerdefrei.

Graphästhesie In einer Schweizer Studie wurden 40 Rückenschmerzpatienten mit Bewegungskontroll- und Graphästhesie-Übungen therapiert [8] ( Abb. 3). Die Patienten bewerteten anhand des Roland-Morris-Fragebogens die Verbesserung ihrer täglich empfundenen Behinderung am Ende mit ca. 40\%. Im Vergleich mit einer ähnlich gestalteten Fallserien-Studie mit gleichen Inhalten und Zielen, jedoch ohne Graphästhesie-Übungen, waren die Resultate gleichartig [14].

Trainingsaufbau Prinzipiell sollte jedes Übungsprogramm so aufgebaut sein, dass in jedweder Phase die Voraussetzung für die nächsthöhere Stufe geschaffen wird. Für das Graphästhesie-Training bedeutet dies etwa eine Veränderung des Berührungs-Stimulus. Die Reizsetzung erfolgt dann klein- statt großflächig, spitz oder stumpf etc. Oder aber die Intention der taktilen Stimuli ist anders geartet, und der Patient soll anstatt Berührungspunkte zu identifizieren, nun Buchstaben oder Wörter auf seinem Rücken lesen oder Rechnungen ausführen.

Für jede Trainingsphase sind zwei Wochen vorgesehen. Können die Aufgaben nicht erfüllt werden, wird die Phasendauer wochenweise verlängert. Sollten Bedenken des Patienten auftreten oder Flare-Ups imponieren, wird der Schwierigkeitsgrad der Übung reduziert oder der Fokus weg vom betroffenen Körperteil gelenkt.

Eine Zusammenfassung, wie das Training zur Verbesserung der Körperwahrnehmung stufenweise gesteigert werden kann, ist nachfolgender Tabelle zu entnehmen ( $\triangleright$ Tab. 2).

\section{Motor Imagery}

Mittels regelmäßig durchgeführter Motor Imagery können u.a. Qualität, Quantität und Timing einer Bewegung verbessert werden. Angewandt wird die Bewegungsvorstellung beispielsweise zur Leistungssteigerung im Sport [4, 5]. In der Physiotherapie liegt der Fokus des mentalen Trainings dagegen auf der Rückführung kortikaler Veränderungen, die als Treiber für chronische Rückenschmerzen identifiziert wurden [4].

Bewegungsvorstellung Bei der Motor Imagery wird zwischen kinästhetischer und visueller Bewegungsvorstellung unterschieden. Die kinästhetische Bewegungsvorstellung konzentriert sich auf das Anfühlen einer Bewegung. Dabei wird der primär sensomotorische Kortex mehr aktiviert, wenn sich das Anfühlen der Bewegung egozentrisch auf die eigene als allozentrisch auf eine andere Person bezieht [13]. Im Gegensatz hierzu beobachtet die visuelle Bewegungsvorstellung eine Bewegung von außen.

Sowohl bei der Beobachtung einer Bewegung als auch bei deren Ausführung sind Spiegelneurone aktiv [24]. Diese unterstützten das motorische Lernen durch Imitation [9, $12,13]$.

\section{Isolierte Low-Load-Anspannung lokaler Muskeln}

\section{M. transversus abdominis}

Für das Training des M. transversus abdominis (MTA) sollte der Patient in einem ruhigen Raum möglichst entspannt in Rücken- oder Seitlage auf der Behandlungsbank gelagert werden. Der Bauch zwischen Symphyse und unterem Rippenbogen muss zur Palpation und Beobachtung der Atmungsexkursion frei sein. Mittels Ultraschallgerät kann der Tonus des querverlaufenden Bauchmuskels vom Therapeuten genauer überprüft und vom Patienten visualisiert werden. Dabei hat sich gezeigt, dass das Erlernen einer isolierten, kompensationsfreien LowLoad-Anspannung des MTA unter visueller Kontrolle eines Ultraschallgeräts für die meisten Patienten am praktikabelsten ist.

Wortwahl Für den Behandlungserfolg ist die Wahl der Worte bei einer therapeutischen Anweisung entscheidend. Aufforderungen wie „Ziehen Sie Ihren Bauch ein!“ oder „Ziehen Sie den Bauchnabel zur Wirbelsäule!“ resultieren oftmals in einem überschnellen und zu starken Anspannen des MTA. Dieses wiederum führt dann zum kompensatorischen Anspannen der globalen Bauchmuskulatur. Um dem Patienten diese Kompensation zu veranschaulichen, soll dieser leicht hüsteln. Via Ultraschall wird deutlich, dass beim Hüsteln nicht allein der MTA ruckartig anspannt, sondern gleichzeitig auch die schrägen Bauchmuskeln kontrahieren. 
- Tab. 2 Zusammenfassung eines sensorisch und motorisch ausgerichteten Trainingsprogramms zur Verbesserung der Körperwahrnehmung (nach Wand et al. 2011) [27].

\begin{tabular}{|c|c|c|}
\hline Phase & Sensorisches Training & Motorisches Training \\
\hline \multirow[t]{2}{*}{1} & Lokalisation: & Erkennen der Seite: \\
\hline & $\begin{array}{l}\text { - Ort des Stimulus } \\
\text { - 1. Woche: mit visuellem Feedback } \\
\text { - 2. Woche: ohne visuelles Feedback }\end{array}$ & $\begin{array}{l}\text { " „Recognise“-Software } \\
\text { - Erkennen von linker/rechter Seite des Rückens } \\
\text { - Progression: } \\
\text { - kürzere Zeit der Präsentation }\end{array}$ \\
\hline \multirow[t]{2}{*}{2} & Lokalisation und Art des Stimulus: & Imitation von gezeigten Bewegungen: \\
\hline & $\begin{array}{l}\text { - Ort des Stimulus } \\
\text { - Art des Stimulus: } \\
\text { - klein vs. groß } \\
\text { - spitz vs. stumpf } \\
\text { - Stimuli an mehreren Stellen am Rücken }\end{array}$ & $\begin{array}{l}\text { - Betrachtung eines Videos mit einem sich bewegenden } \\
\text { Model und Nachahmung der Bewegung } \\
\text { - 1. Woche: kleine Bewegungsamplitude } \\
\text { - 2. Woche: volles Bewegungsausmaß }\end{array}$ \\
\hline \multirow[t]{2}{*}{3} & Graphästhesie 1: & Isometrische Low-load-Rekrutierung lokaler Muskeln: \\
\hline & $\begin{array}{l}\text { - Erkennen von Zahlen und Buchstaben } \\
\text { - Progression: } \\
\text { - Größe } \\
\text { - Orientierung } \\
\text { - Geschwindigkeit }\end{array}$ & $\begin{array}{l}\text { - M. transversus abdominis } \\
\text { - Mm. multifidi, tiefe Schicht } \\
\text { - Kokontraktion mit dem Beckenboden } \\
\text { - Kombination mit Dissoziationsübungen }\end{array}$ \\
\hline \multirow[t]{2}{*}{4} & Graphästhesie 2: & Kleine Bewegungen mit maximalem Feedback: \\
\hline & $\begin{array}{l}\text { - Erkennen von Wörtern mit drei Buchstaben } \\
\text { - Progression: } \\
\text { - Größe } \\
\text { - Orientierung } \\
\text { - Geschwindigkeit } \\
\text { - Überlappung der Buchstaben }\end{array}$ & $\begin{array}{l}\text { - Spiegel zur visuellen Kontrolle } \\
\text { - intersegmentale Selbst-Palpation zur taktilen Kontrolle } \\
\text { - elastisches Tape: Palpation } \\
\text { - Repositions-Training } \\
\text { - Bewegungen entsprechend Anweisung }\end{array}$ \\
\hline \multirow[t]{2}{*}{5} & Graphästhesie 3: & Volle Bewegungen mit maximalem Feedback: \\
\hline & $\begin{array}{l}\text { - Berechnen einfacher Summen } \\
\text { - Progression: } \\
\text { - Größe } \\
\text { - Orientierung } \\
\text { - Geschwindigkeit } \\
\text { - Überlappung der Zahlen }\end{array}$ & $\begin{array}{l}\text { - Spiegel zur visuellen Kontrolle } \\
\text { - intersegmentale Selbst-Palpation zur taktilen Kontrolle } \\
\text { - elastisches Tape: Palpation } \\
\text { - Repositions-Training } \\
\text { - Bewegungen entsprechend Anweisung }\end{array}$ \\
\hline
\end{tabular}

Entsprechend sollen Anweisungen an den Patienten durch die Vermittlung von Bildern erfolgen. Formulierungen wie „Spannen Sie Ihren Bauch an, als ob Sie den Reißverschluss Ihrer Jeans zuziehen!“ oder „Spannen Sie Ihren Bauch an, als ob Sie Ihre vorderen Beckenspitzen [SIAS] zur Mitte ziehen!“ veranschaulichen das Übungsziel.

\section{M. multifidus}

Das isolierte Anspannen der Mm. multifidi erfolgt aus der Bauchlage. Der Therapeut legt seinen Zeige- und Mittelfinger im Pinzettengriff beidseits dicht an den Dornfortsatz eines lumbalen Wirbels an. Der Patient soll nun an dieser Stelle den Muskel langsam und leicht anspannen.
Visualisierung Um eine Multifidus-Spannung zu generieren, eignen sich Anleitungen wie: „Machen Sie ein leichtes Hohlkreuz!“ oder „Stellen Sie sich vor, Ihr Kreuzbein ist ihr Schwanz wie bei einem Hund, und Sie wollen diesen Schwanz nun heben“. Falls die Anweisung vom Patienten umgesetzt werden kann, soll dieser die Muskelspannung nun ohne Bewegung realisieren. Auf diesem Weg wird aus einer „globalen“ eine „lokale“ Aktivität.

Wahrnehmungsübung Um das Gefühl einer Multifidus-Spannung zu vermitteln, eignet sich folgende Wahrnehmungsübung im Stand: Die Hände des Patienten liegen beidseits paravertebral auf Höhe von L5. Anschließend macht der Patient einen Schritt nach vorne und spürt die sofortige Kontraktion des M. multifidus. Im nächsten Zug versucht er, die lokale Muskelanspannung zu reproduzieren, ohne einen Schritt zu machen. Ziel der Übung ist, die Multifidus-Spannung so klein wie möglich, dabei aber noch fühlbar zu halten: „Je weniger, desto besser.“ 

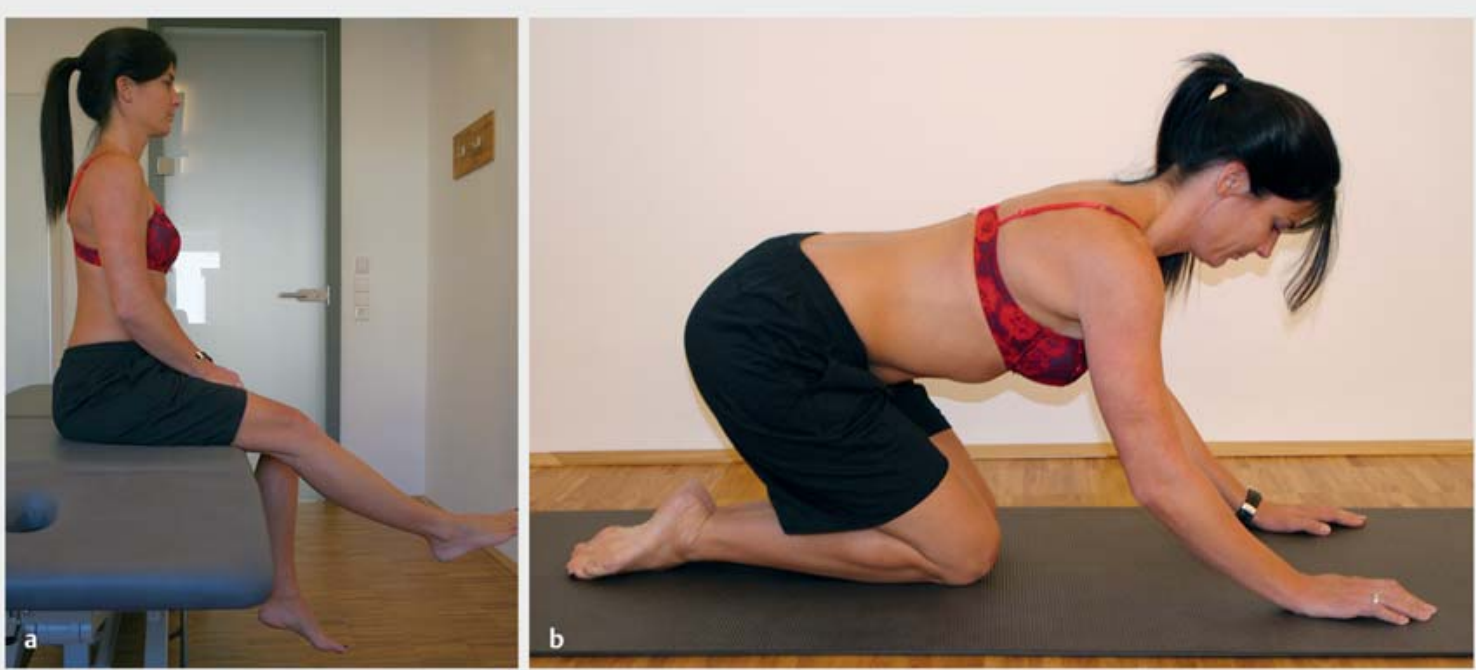

Abb. 4 Dissoziative Übungen. Quelle: Thieme Gruppe

a Die Wirbelsäule soll während der Bewegungen in den Hüft- und/oder anderen Beingelenken in Neutralstellung gehalten werden. b Bei der Verschiebung im Vierfüßler soll das Becken nach hinten-unten bzw. vorn verschoben werden, ohne dass die Wirbelsäule flektiert bzw. extendiert.

\section{Dissoziative Übungen}

Wahrnehmungsübungen Spezielle Bewegungen sind nur dann „echte“ Wahrnehmungsübungen, wenn der Fokus des wachen und motivierten Patienten allein auf der Wahrnehmung liegt. Er soll spüren, wo und wie eine Bewegung stattfindet und welche Muskeln sich hierfür anund entspannen. Auf Grund der übungsbedingten schnellen mentalen Ermüdung des Patienten wird die Übungszeit kurz, die Übungsfrequenz aber hoch gewählt. Folglich soll mehrmals täglich geübt werden.

Dissoziatives Üben bedeutet das bewusste Bewegen von Körperabschnitten unabhängig voneinander. Folglich sollen weiterlaufende Bewegungen angrenzender Körperabschnitte aktiv und bewusst verhindert werden. Als praktische Übung soll der Patient eine Hüft-Flexion ohne weiterlaufende LWS-Flexion durchführen oder sein Becken samt LWS still halten, während er die BWS in Flexion, Extension oder Rotation bewegt ( $\triangleright$ Abb. 4).

\section{Zusammenfassung}

Die aktuelle Studienlage liefert deutliche Indizien, dass chronische Schmerzen die Körperwahrnehmung beeinträchtigen können. Unklar bleibt, wie stark die Wahrnehmungsstörungen ausgeprägt sind. Dessen ungeachtet kann die Körperwahrnehmung eines Patienten zuverlässig auf verschiedene Arten getestet und somit auch deren Störung erfasst werden. Für die Therapie stehen unterschiedliche Möglichkeiten bereit. Die Effektivität der vorgestellten Therapieform wurde bei CRPS-Patienten am besten nachgewiesen. Bei Rückenschmerzen ist die Evidenz der Effektivität weiterhin unklar, diesbezügliche Studien müssen folgen.

\section{Autorinnen/Autoren}

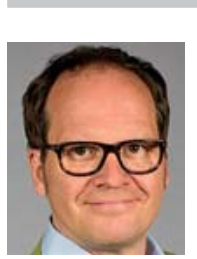

\section{Prof. Dr. Hannu Luomajoki}

ist Physiotherapeut und leitet das Masterprogramm muskuloskeletale Physiotherapie an der ZHAW Winterthur. Er ist Mitglied der NOI (Neuroorthopädisches Institut, „ButlerGruppe") und forscht unter anderem zu den Themen unspezifische Kreuzschmerzen und Chronifizierungsrisiko bei Schmerzpatienten.

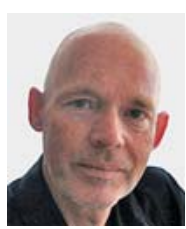

\section{Fritz Zahnd}

ist Physiotherapeut (OMT) und Dozent in einem Master-Programm für muskuloskeletale Physiotherapie an der Fachhochschule Winterthur. Er ist Mitbegründer des Schweizerischen Verbandes für Orthopädische Manipulative Physiotherapie (SVOMP) und publiziert in mehreren Fachbüchern und Zeitschriften zur Manuellen Therapie und Trainingstherapie.

Korrespondenzadresse

Prof. Dr. PD Hannu Luomajoki

Zürcher Hochschule für Angewandte Wissenschaften ZHAW Technikumstr. 71 $\mathrm{CH}-8400$ Winterthur luom@zhaw.ch 


\section{Korrespondenzadresse}

\section{Fritz Zahnd}

Bühlstrasse 10

CH-8125 Zollikerberg

fzahnd@bluewin.ch

Literatur

[1] Acerra NE, Moseley GL. Dysynchiria: watching the mirror image of the unaffected limb elicits pain on the affected side. Neurology 2005; 65: 751-753

[2] Bray H, Moseley GL. Disrupted Working Body Schema Of The Trunk In People With Back Pain. British journal of sports medicine. Br J Sports Med 2011 Mar; 45: 168-173. doi:10.1136/ bjsm.2009.061978

[3] Catley M], Tabor A, Wand BM et al. Assessing tactile acuity in rheumatology and musculoskeletal medicine - how reliable are two-point discrimination tests at the neck, hand, back and foot? Rheumatology (Oxford) 2013; 52: 1454-1461. doi:10.1093/rheumatology/ket140

[4] Daffada P, Walsh N, McCabe C et al. The impact of cortical remapping interventions on pain and disability in chronic low back pain: A systematic review. Physiotherapy 2015; 101: 2533. doi:10.1016/j.physio.2014.07

[5] Dettmers C, Nedelko V. Mentales Training. Neuroreha 2011: 24-31. doi:10.1055/s-0031-1273064

[6] Ehrenbrusthoff K, Ryan CG, Grüneberg C et al. The German Version of the Fremantle Back Awareness Questionnaire (FreBaQ) -Transcultural adaptation and preliminary practicability findings. Im Internet: https://www.physio-deutschland. de/fileadmin/data/bund/events/BuKo2014/Pr\%C3\%A4 sentationen/PDF_Poster_Ehrenbrusthoff_Katja.pdf; Stand: 25.03.2018

[7] Flor $\mathrm{H}$. The functional organization of the brain in chronic pain. Prog Brain Res 2000; 129: 313-322. doi:10.1016/50079-6123 (00)29023-7

[8] Flor $\mathrm{H}$, Birbaumer N. Phantom limb pain: cortical plasticity and novel therapeutic approaches. Curr Opin Anaesthesiol 2000; 13: $561-564$

[9] Gutknecht M, Mannig A, Waldvogel A et al. The effect of motor control and tactile acuity training on patients with non-specific low back pain and movement control impairment. J Bodyw Mov Ther 2015; 19: 722-731. doi:10.1016/j. jbmt.2014.12.003

[10] Grunwald M, Beyer L, Hrsg. Der bewegte Sinn: Grundlagen und Anwendungen zur haptischen Wahrnehmung. Basel: Springer 2013

[11] Hari R, Forss N. Magnetoencephalography in the study of human somatosensory cortical processing. Philos Trans R Soc Lond B Biol Sci 1999; 354: 1145-1154. doi:10.1098/ rstb. 1999.0470

[12] lacoboni M. Neural mechanisms of imitation. Curr Opin Neurobiol 2005; 15: 632-637. doi:10.1016/j.conb.2005. 10.010

[13] Jackson PL, Meltzoff AN, Decety J. Neural circuits involved in imitation and perspective-taking. Neuroimage 2006; 31: 429439. doi $0.1016 / j$.neuroimage.2005.11.026
[14] Luomajoki H, Kool J, De Bruin ED et al. Improvement in low back movement control, decreased pain and disability, resulting from specific exercise intervention. Sports Med Arthrosc Rehabil Ther Technol 2010; 23: 11. doi:10.1186/1758-25552-11

[15] Luomajoki H, Moseley GL. Tactile acuity and lumbopelvic motor control in patients with back pain and healthy controls. Br J Sports Med 2011; 45: 437-440. doi:10.1136/bjsm. 2009.060731.

[16] Moberg E. Surgical rehabilitation of the upper limb in tetraplegia. Paraplegia 1990; 28: 330-334. doi:10.1038/sc. 1990.43

[17] Moseley GL. Graded motor imagery for pathologic pain: a randomized controlled trial. Neurology 2006; 67: 21292134. doi:10.1212/01.wnl.0000249112.56935.32

[18] Moseley GL. Graded motor imagery is effective for longstanding complex regional pain syndrome: a randomised controlled trial. Pain 2004; 108(1-2): 192-198. doi:10.1016/ j.pain.2004.01.006

[19] Moseley GL. I can't find it! Distorted body image and tactile dysfunction in patients with chronic back pain. Pain 2008; 140: 239-243. doi:10.1016/j.pain.2008.08.001.

[20] Moseley GL, Zalucki NM, Wiech K. Tactile discrimination, but not tactile stimulation alone, reduces chronic limb pain. Pain 2008; 137: 600-608. doi:10.1016/j.pain.2007.10.021

[21] Pleger B, Ragert P, Schwenkreis P et al. Patterns of cortical reorganization parallel impaired tactile discrimination and pain intensity in complex regional pain syndrome. Neuroimage 2006; 32: 503-510. doi:10.1016/j.neuroimage.2006.03.045

[22] Pleger B, Tegenthoff M, Ragert et al. Sensorimotor retuning [corrected] in complex regional pain syndrome parallels pain reduction. Ann Neurol 2005; 57: 425-429. doi:10.1002/ ana.20394

[23] Ramachandran VS. Plasticity in brain development. Sci Am 1989; 260: 8-9

[24] Rizzolatti G, Fadiga L, Gallese V et al. Premotor cortex and the recognition of motor actions. Brain Res Cogn Brain Res 1996; 3: $131-341$

[25] Schmid AB, Coppieters MW. Left/right judgment of body parts is selectively impaired in patients with unilateral carpal tunnel syndrome. Clin J Pain 2012; 28:615-622. doi:10.1097/AJP.0b013e31823e16b9

[26] Wand BM, James M, Abbaszadeh S et al. Assessing self-perception in patients with chronic low back pain: development of a back-specific body-perception questionnaire. J Back Musculoskelet Rehabil 2014; 27: 463-473. doi:10.3233/BMR140467

[27] Wand BM, O'Connell NE, Di Pietro F et al. Managing chronic nonspecific low back pain with a sensorimotor retraining approach: exploratory multiple-baseline study of 3 participants. Physical therapy 2011; 91: 535-546. doi:10.2522/ptj. 20100150

Bibliografie

DOI https://doi.org/10.1055/a-0579-2136

Der Schmerzpatient 2018; 1: 123-129

(c) Georg Thieme Verlag KG Stuttgart · New York ISSN 2512-6210 